\title{
Axel Görlitz
}

Demokratie im Wandel 
Axel Görlitz

\section{Demokratie im Wandel}

(W)

Westdeutscher Verlag

Köln und Opladen 1969 
ISBN 978-3-322-97981-0

ISBN 978-3-322-98568-2 (eBook)

DOI 10.1007/978-3-322-98568-2

Verlags-Nr. 054602

(C) 1969 by Westdeutscher Verlag $\mathrm{GmbH}$, Köln und Opladen Gesamtherstellung: Druckerei Dr. Friedrich Middelhauve GmbH, Opladen Umschlag: Hanswerner Klein, Opladen 


\section{Zum Geleit}

Ob die rechtsstaatliche Demokratie nur einem Wandlungsprozeß unterliegt, dessen Gründe und Verlauf feststellbar sind, oder ob sie sich in einer Krise befindet, deren Ende weder zeitlich noch inhaltlich absehbar ist, ist eine der entscheidenden Fragen unserer Zeit - vielleicht die entscheidende schlechthin. Unstrittig sollte sein, daß die Demokratie der Gegenwart ein Dreifaches leisten müßte, wenn sie und die mit ihr verbundenen Werte auch in Zukunft bestehen sollen.

$\mathrm{Da}$ ist zum ersten das notwendige Aufarbeiten der Vergangenheit: Verfassungstext, politische Vorstellungen und Verhaltensweisen sind weithin von den Begriffen des 19. Jahrhunderts geprägt. Diese aber entsprechen den politischen und sozialen Bedingungen jener Zeit. Da sich die Bedingungen geändert haben, sind die Begriffe funktionslos geworden oder sie dienen nur noch zur Verhüllung. Insofern geht es zunächst um eine Revision von Begriffen und dann um eine Reform der Demokratie, die sich wiederum in eine Fülle von Teilaufgaben zerlegen läßt, weil eben von der Reform des Parlaments, der Verwaltung, der Finanzverfassung, des öffentlichen Dienstes, der Regierungsstruktur und der unendlich vielen Verfahrensweisen die Rede sein muß - und nicht nur die Rede!

Zum zweiten ist dieser Demokratie gleichzeitig angesonnen, der Veränderungen Herr zu werden, die in der Gegenwart sichtbar sind und in ihrem Verlauf unsere Zukunft bestimmen werden. Keine Generation zuvor hat so viel von derartigen Entwicklungen gewußt. Keiner war mithin die Zukunft so unmittelbar als Aufgabe gestellt. Keine war sich dessen so sicher, daß ein bloßes Weitermachen Rückschritt, ja unmittelbare Gefährdung bedeutet. Infolgedessen muß auch von den Veränderungen, von der künftigen Raumordnung, vom weiteren Prozeß der Urbanisierung, von der vermehrten Freizeit, von der immer noch zunehmenden internationalen Interdependenz und von den Gefahren in aller Welt, unter denen die Kriegsgefahr nicht einmal die größte ist, die Rede sein - und nicht nur die Rede!

Zum dritten endlich kann man bei alldem nicht der Einsicht ausweichen, daß die moderne Demokratie zum Untergang verurteilt ist, wenn sie nicht wirklich soziale Demokratie ist - mit allen Konsequenzen. Die ständig noch anwachsenden ,öffentlichen“ Aufgaben lassen auch die Struktur der Gesellschaft zur genuin politischen Aufgabe werden. Politik muß zukunftsorientiert sein. Sie ist es aber nur dort, wo sie selbst ein Bild von 
der künftigen Gesellschaft entwirft, wo sie Freiheit nicht nur formal schützt, sondern material ermöglicht, wo ihr der Mensch „,heilig“ ist, nicht aber die Zufälligkeit oder die Geschichtlichkeit gesellschaftlicher Umstände. Sinnvolle Raumordnung kann das Eigentum am Boden, sinnvolle Wirtschaftsstrukturpolitik kann das Kapitaleigentum nicht unangetastet lassen, und sinnvolle Bildungspolitik, die der „Bildung“ des Menschen und nicht nur seiner gesellschaftlichen Brauchbarkeit dient, kann nicht die alten Gegensätze zwischen höherer und niederer Bildung, zwischen Akademikern und Nichtakademikern verewigen und in der tradierten Weise gesellschaftlich zum Ausdruck bringen wollen. Unsere Möglichkeiten reichen aus, um die Gesellschaft, wie sie ist, auf die hin zu verändern, die wir uns wünschen, wobei es uns nicht gestattet ist zu wünschen, es möge alles so bleiben. Dennoch ist der Spielraum groß. Der eigentliche Konflikt hier in diesem Lande entzündet sich wie in den Vereinigten Staaten oder in Frankreich an dem Bild von der künftigen Gesellschaft, gleichgültig ob das die rebellierenden Studenten, die unruhigen Bauern, die ängstlichen Handwerker rational zum Ausdruck bringen oder nicht. Und dann stellt sich eben die Frage, ob und wie die Demokratie dazu tauglich gemacht werden kann, auch diesen Konflikt auszutragen. „Tauglich gemacht werden kann!“ - sie ist es nicht. Die Geister scheiden sich nur daran, daß die einen die Reformfähigkeit des Systems unterstellen, während die anderen sie bestreiten.

Es gehört Mut dazu, in dieser geschichtlichen Situation über die spezifische Fragestellung, wie sie der Wissenschaft in der Regel eigentümlich ist, hinauszugehen und gleich nach der „Demokratie im Wandel“" zu fragen, was dann gleichzeitig bedeutet, dem Stand der Kenntnisse und Einsichten oder Theorien in den verschiedenen, hier beteiligten Wissenschaften ihr Recht zu geben. Denn zu alldem hat der Soziologe, der Historiker, der Politikwissenschaftler, der Jurist, der Psychologe, der Sozialpolitiker oder auch der Philosoph und der Anthropologe etwas zu sagen - und er hat es gesagt. So gilt es auszuwählen, zu referieren, den Zusammenhang herzustellen und die eigene Meinung zu begründen. $\mathrm{Zu}$ beurteilen, ob das in dem vorliegenden Versuch gelungen ist, muß dem Leser überlassen bleiben. Der Leser wird finden, daß die Probleme weniger in ihrer praktischen Evidenz und mehr abstrahierend analytisch angegangen sind. Er wird das pädagogische Bemühen des Verfassers verspüren, das sich im sozialpsychologischen Schlußteil und in den Anregungen für die politische Bildungsarbeit noch einmal verdichtet. Dabei ist freilich zu fragen, ob es didaktisch zweckmäßig ist, daß mehr von der politischen Ordnung der Gesellschaft und den in dieser Ordnung angesiedelten Verfahren gesprochen wird und weniger von den gegenwärtigen und künftigen Aufgaben der Politik, weil den Verfasser mehr das ,,wie“ der Politik interessiert als das ,was“. Das mag dem ungenügend erscheinen, der das ,,wie“ und das ,was" nicht voneinander trennen will und beides nur durch einander vermittelt ansieht. Es macht zugleich verständlich, daß der Verfas- 
ser die Wandlungen beschreibt und erklärt und oft mit dem Erklären sich begnügt. Darf aber mehr verlangt werden, wenn die Aufgabe in der Analyse und nicht in der Prognose und nicht im Entwurf der Theorie einer künftigen Gesellschaft gesehen wird? $\mathrm{Zu}$ messen ist ein solcher Versuch am eigenen Anspruch. Er ist vielleicht bescheiden, weil er sich eben auf die Analyse beschränkt. Freilich muß man dem hinzufügen, daß hierzulande nicht einmal die Analyse wirklich zur Kenntnis genommen wird. Der Aufklärungsprozeß beginnt erst. Ob er durch die mancherlei Schwierigkeiten, denen man sich etwas unvermutet seit einiger Zeit auch in der Bundesrepublik gegenübersieht, erleichtert oder erschwert wird, weiß niemand. Das darf den Wissenschaftler aber nicht hindern, unverdrossen zu sagen, was er erarbeitet hat und zu wissen meint. Und es darf ihn auch nicht hindern zu hoffen, daß er die Leser findet, an die er gedacht hat. Ich kann dem Verfasser nur wünschen, daß sich diese Hoffnung erfüllt.

Thomas Ellwein 


\section{Inhaltsverzeichnis}

Vorwort $\ldots \ldots \ldots \ldots \ldots \ldots \ldots \ldots \ldots \ldots \ldots \ldots \ldots \ldots \ldots \ldots \ldots \ldots$

A. Demokratie und Verfassung $\ldots \ldots \ldots \ldots \ldots \ldots \ldots \ldots$

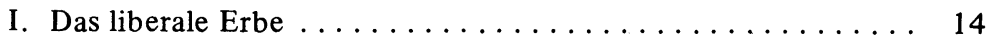

1. Der bürgerliche Nationalstaat $\ldots \ldots \ldots \ldots \ldots \ldots \ldots 16$

2. Der liberale Rechtsstaat $\ldots \ldots \ldots \ldots \ldots \ldots \ldots \ldots \ldots \ldots \ldots$

II. Strukturprobleme der Bundesrepublik $\ldots \ldots \ldots \ldots \ldots 26$

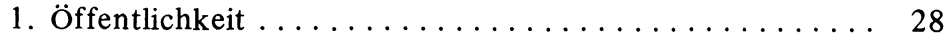

2. Parteien und Verbände ................ 37

3. Wahlen ........................ 46

4. Parlament ..................... 56



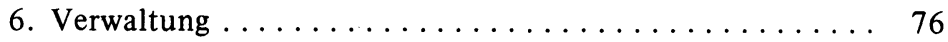

7. Gerichtsbarkeit .................. 86

B. Demokratie und moderner Staat $\ldots \ldots \ldots \ldots \ldots \ldots \ldots \ldots$

I. Politik und Technik . . . . . . . . . . . . . . . . . 101

1. Technokratie ........................ 104

2. Planung . . . . . . . . . . . . . . . 113

II. Politik und Wissenschaft $\ldots \ldots \ldots \ldots \ldots \ldots \ldots \ldots \ldots$

C. Demokratie und Staatsbürger $\ldots \ldots \ldots \ldots \ldots \ldots \ldots \ldots \ldots \ldots$

I. Der Deutsche . . . . . . . . . . . . . . . . . . . . . 135

1. Das Bild des Bürgers . . . . . . . . . . . . . . 136

2. Das Modell des Bürgers ................. 145

II. Politische Bildung $\ldots \ldots \ldots \ldots \ldots \ldots \ldots \ldots \ldots \ldots \ldots \ldots$

1. Psychologische Probleme . . . . . . . . . . . . . . 157

2. Sozialpsychologische Probleme .............. 162

3. Didaktische Probleme ................. 169

Literaturverzeichnis ........................ 179

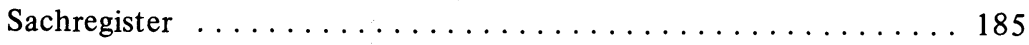




\section{Vorwort}

Die Behauptung, Opas Demokratie sei tot, artikuliert ein Unbehagen an den tradierten Institutionen, das sich nicht mit dem Hinweis auf ein prosperierendes sogenanntes demokratisches Regierungssystem aus der Welt schaffen läßt. In der Tat sind Begriffe wie Öffentlichkeit, Konkurrenzprinzip oder Gleichheit heute überall fragwürdiger denn je, nicht zuletzt weil sie ihren Kampfcharakter eingebüßt haben und in systemstabilisierende Werte umfunktioniert wurden. Stabilität verwandelt sich leicht in Stagnation, und am gesellschaftlichen Stillstand entzündet sich der Protest der Revolutionäre, die sich mit einem nur technischen oder kulturellen Fortschritt nicht bescheiden wollen. Sie setzen der jeweiligen strukturverschleiernden Ideologie ihre Utopie entgegen, die um so mehr Sprengkraft entwickelt, je starrer die Institutionen sind. Ein System droht jedenfalls dann zu erstarren, wenn es seine überlieferten Axiome nicht mehr reflektiert, vielmehr jeden Reflexionsversuch mit den tradierten Werten übertönt, die es von einer Art tibetanischer Gebetsmühlen ständig herunterklappern läßt.

Dieses Buch ist ein Reflexionsversuch mehr. Es sucht eine Antwort auf die Frage, ob die überkommenen demokratischen Werte und Institutionen für den modernen Staat etwas taugen. Als Ansatz der Überlegungen dient der Öffentlichkeitsbegriff, der mir eine zentrale institutionelle Kategorie des demokratischen Systems zu sein scheint. Ich meine, die Erörterungen verdeutlichen, daß die Demokratie zwar nicht stirbt, wohl aber kränkelt. Die Therapie, die hier vorgeschlagen wird, kuriert bestenfalls einige Symptome, dagegen sicher nicht die ganze Krankheit; ein erster diagnostischer Versuch wird eben nur selten zu einer umfassenden Therapie führen. Das gilt um so mehr, als eine Reform der institutionellen und normativen Bedingungen wenig nützt, solange sich die Menschen, die in diesem System leben, anderen Institutionen und Normen verbunden fühlen. Auch der noch so demokratisch organisierte Staat hilft wenig, wenn die Gesellschaft nicht demokratisiert ist. Die Demokratie kurieren heißt zwei Patienten behandeln, nämlich Staat und Bürger.

Insofern versteht sich dieses Buch auch als Beitrag zur politischen Bildung. Es ist damit an ein unbestimmtes Publikum adressiert, und ich habe versucht, es auf einen möglichst großen gemeinsamen sprachlichen Nenner zu bringen. Da Vereinfachungen häufig verfälschen, ließen sich allerdings terminologische Schwierigkeiten nicht immer umgehen. Leser, 
die mit dem politologischen, soziologischen oder psychologischen Sprachgebrauch nicht vertraut sind, bitte ich um Geduld; in der Regel werden Fachbegriffe erklärt, sobald eine Erklärung angemessen erscheint. So findet sich etwa zu Beginn des Abschnitts B eine Einführung in die soziologische Terminologie, und wer mit Begriffen wie Rolle oder Institution wenig anzufangen weiß, mag die Lektüre dieses Kapitels vorziehen. Schlimmstenfalls muß Verständnisschwierigkeiten die Lektüre ausräumen, auf die zu Anfang jedes Kapitels verwiesen wird.

Diese Lektüre ist in dem jeweiligen Kapitel verarbeitet. Das Bekenntnis, daß ihre Auswahl subjektiv ist, wäre müßig: Die Äußerungen zu den Themen, die in diesem Buch angeschnitten werden, füllen Bibliotheken, und niemand dürfte noch imstande sein, eine derartige Papierflut zu bewältigen. Die Nichtbeachtung eines Werkes bedeutet mithin keinesfalls seine Abwertung. Im übrigen möchten die Literaturbelege zwei Herren dienen. Im Interesse der Leser, die ein billiges Buch wollen, wurde auf Fußnoten verzichtet, die den Satz und damit den Preis verteuert hätten. Lesern, die genauere Literaturnachweise wünschen, sollen Zitate und Namensnennungen im Text Hinweise geben, auf welche Werke sich die jeweiligen Erörterungen stützen.

Axel Görlitz 